Fisioter Bras 2021;22(2);261-271

doi: $10.33233 / \mathrm{fb} . v 22 \mathrm{i} 2.4288$

\title{
REVISÃO
}

\section{A importância da reabilitação pulmonar em pacientes com COVID-19}

The importance of pulmonary rehabilitation in patients with COVID-19

Paula Lima Bosi*, Luciana Fernanda de Freitas Januzzi*, Priscila Barreto de Paula*, Camila Carvalho de Oliveira*, Claudio Alvin Scianni*, Thais Aparecida Nunes da Costa*, Claudia Teixeira Monteiro de Freitas Teixeira*, Raphael Borges de Oliveira Gomes*, Adriano Santos Clovis*, Giselle Aline dos Santos Gonçalves*, Paulo Henrique Silva Maia, D.Sc. **

*Docente do curso de Fisioterapia da Faculdade Pitágoras, Betim, MG, Brasil, **Enfermeiro, Doutor em Saúde Coletiva pela UFMG

Recebido em 28 de agosto de 2020; aceito em 16 de abril de 2021.

Correspondência: Paula Lima Bosi, Rua Níquel 68, 1102 Serra 30220-280 Belo Horizonte MG

\author{
Paula Lima Bosi: plimab@gmail.com \\ Luciana Fernandes Freitas Januzzi: Iufjanuzzi@gmail.com \\ Priscila Barreto de Paula: pricala@uol.com.br \\ Camila Carvalho de Oliveira: camila.carvalho@kroton.com.br \\ Claudio Alvin Scianni: claudio.scianni@kroton.com.br \\ Thais Aparecida Nunes da Costa: thais.nunes@kroton.com.br \\ Claudia Teixeira Monteiro de Freitas Teixeira:claudia.t.teixeira@kroton.com.br \\ Raphael Borges de Oliveira Gomes: raphaelbogomes@gmail.com \\ Adriano Santos Clovis: adriano.cloves@kroton.com.br \\ Giselle Aline dos Santos Gonçalves: giselle.goncalves@kroton.com.br \\ Paulo Henrique Silva Maia: caremaia@gmail.com
}

\section{Resumo}

Introdução: Uma doença altamente infecciosa do trato respiratório, a doença de coronavírus 2019 (COVID-19) pode causar disfunção respiratória, física e psicológica em pacientes. Portanto, a reabilitação pulmonar é crucial para pacientes admitidos e que recebem alta da COVID-19. As sequelas de longo prazo são desconhecidas, mas as evidências de surtos anteriores de CoV demonstram comprometimento da função pulmonar e física, redução da qualidade de vida e sofrimento emocional. Métodos: Trata-se de uma revisão sistemática da literatura realizada através de busca digital em 
artigos publicados em revistas impressas e eletrônicas, ensaios clínicos, estudos randomizados, revisões sistemáticas, no período compreendido entre os anos de 2003 e 2020. Resultados: Muitos sobreviventes da COVID-19 que necessitam de cuidados críticos podem desenvolver comprometimentos psicológicos, físicos e cognitivos. Conclusão: Existe uma clara necessidade de orientação sobre a reabilitação dos sobreviventes da COVID-19.

Palavras-chave: infecções por coronavírus; betacoronavírus; medicina física e de reabilitação

\begin{abstract}
Introduction: A highly infectious disease of the respiratory tract, coronavirus disease 2019 (COVID-19) can cause respiratory, physical, and psychological dysfunction in patients. Therefore, pulmonary rehabilitation is crucial for patients admitted and discharged from COVID-19. The long-term sequelae of COVID-19 are unknown, but evidence of previous CoV outbreaks demonstrates impaired lung and physical function, reduced quality of life and emotional distress. Methods: This is a systematic review of the literature carried out through digital bibliographic search of scientific articles published in printed and electronic journals, clinical trials, randomized studies, systematic reviews, in the period between the years 2003 and 2020. Results: Many survivors of COVID-19 that require critical care can develop psychological, physical, and cognitive impairments. Conclusion: There is a clear need for guidance on the rehabilitation of COVID-19 survivors.
\end{abstract}

Keywords: coronavirus infection; betacoronavirus; physical and rehabilitation medicine

\title{
Introducão
}

Em 30 de janeiro de 2020, a Organização Mundial da Saúde (OMS) anunciou a epidemia de 2019-nCoV, uma emergência de saúde pública de interesse internacional. E em 11 de fevereiro de 2020, a OMS nomeou formalmente a doença desencadeada por 2019-nCoV como doença de coronavírus 2019 (COVID-19). No mesmo dia, o grupo de estudo sobre coronavírus do Comitê Internacional de Taxonomia de Vírus nomeou 2019-nCoV como coronavírus 2 da síndrome respiratória aguda grave (SARS-CoV-2) [1]. Ainda em 11 de março de 2020, a OMS elevou o status do novo coronavírus (COVID19) a uma pandemia [1]. Embora as avaliações quanto à pandemia estejam mudando rapidamente, o COVID19 infectou e tirou a vida de milhares de pessoas. Desde que os primeiros casos surgiram, no final de 2019, a COVID19 provocou um caos generalizado nos sistemas de saúde público e privado, que se esforçam para responder 
ao aumento na demanda. Caos provocado também na economia global, pois trabalhadores foram impedidos de trabalhar, e há assim um declínio na economia geral. Constata-se que uma profunda ansiedade está se espalhando quase tão rapidamente quanto a própria doença [2].

O coronavírus é um dos principais patógenos que tem como alvo principal o sistema respiratório humano. O SARS-CoV-2 pertencente à família Coronaviridae, com uma única fita de RNA e um nucleocapsídeo (estrutura composta pelo ácido nucleico do vírus - neste caso RNA - e seu invólucro proteico, o capsídeo) helicoidal. O nome se deve a espículas (estruturas proeminentes) presentes na superfície do vírus, o que lhe dá a aparência de uma coroa solar. As proteínas não estruturais desse vírus desempenham um papel crucial na replicação do vírus, enquanto as proteínas estruturais e auxiliares estão envolvidas na morfogênese e interferem na resposta da imunidade do hospedeiro, respectivamente [3].

Aparentemente o vírus conseguiu fazer a transição de animais para humanos no mercado de frutos do mar de Huanan em Wuhan, China [4]. Os surtos anteriores de coronavírus (CoVs) incluem a síndrome respiratória aguda grave (SARS)-CoV e a síndrome respiratória do Oriente Médio (MERS)-CoV, que foram anteriormente caracterizados como agentes que representam uma grande ameaça à saúde pública [5].

Relevante citar que os casos tendem a ser em aglomerados, que chegam em ondas, e se desenvolvem em surtos maiores em todo o mundo. O primeiro surto documentado ocorreu principalmente em Wuhan [2]. De acordo com o relatório diário da Organização Mundial da Saúde, a epidemia de SARS-CoV-2, até agora registrou 13.876.441 casos confirmados e 593.087 mortes em todo mundo, e no Brasil atingiu 2.012.151 casos com 76.688 mortes, até 18 de julho de 2020 [1]

Com base nas avaliações da OMS e de outras agências de saúde pública, a COVID19 ataca principalmente o sistema respiratório inferior, e o vetor de transmissão parece ser principalmente através de gotículas respiratórias de tosse, espirro e contato próximo [6]. Shi et al. [7] sugeriram que o vírus também pode se espalhar indiretamente através da "contaminação de objetos comuns, aerossolização do vírus em um espaço confinado ou disseminação de pessoas infectadas assintomáticas".

Quanto aos sintomas, a maioria dos pacientes apresenta sintomas leves a moderados. Os sintomas mais comuns são febre, tosse seca, fadiga. Outros sintomas como, faringalgia, dores de cabeça e mialgia também podem ocorrer. Há também relatos descrevendo pacientes com sintomas gastrointestinais, incluindo dor abdominal e diarreia [8]. Aproximadamente $20 \%$ dos pacientes com COVID-19 desenvolvem doença respiratória grave, com uma taxa geral de letalidade de cerca de 2,3\%. Pacientes em estado grave da doença geralmente apresentam febre, tosse seca, 
dispneia e infiltrados pulmonares bilaterais no tórax. As complicações mais comuns do COVID-19 incluem síndrome do desconforto respiratório agudo (SDRA), insuficiência respiratória, lesão hepática, lesão miocárdica aguda, lesão renal aguda, choque séptico e até falência de múltiplos órgãos [9]. Dentre as pesquisas laboratoriais, os estudos apontam para leucopenia e linfopenia os mais comuns [10-12], sendo a linfopenia uma característica fundamental da COVID-19, podendo ser observado pelo aumento do lactato desidrogenase (LDH), da creatina quinase $(\mathrm{CK})$ e aumento nos níveis de marcadores inflamatórios como proteína $\mathrm{C}$ reativa e citocinas pró-inflamatórias.

A manifestação clínica usual após um período de incubação de 4 dias inclui febre, tosse seca e persistente, mialgia e falta de ar. Casos raros, mas com risco de vida, resultam em pneumonia e síndrome do desconforto respiratório agudo (SDRA). De acordo com os Centros de Controle e Prevenção de Doenças (CDC), até 80\% das pessoas infectadas experimentam doenças leves a moderadas, exigindo poucas intervenções, mas as demais serão seriamente afetadas e exigirão cuidados médicos intensivos. Embora tenha dificuldade para estimar com precisão, a OMS relata uma taxa de mortalidade de 3,4\% [13]. Essa estimativa, no entanto, camufla a realidade de que as taxas de mortalidade diferem por idade e, para aqueles com mais de 80 anos, a taxa está mais próxima de $15 \%[14,15]$.

De qualquer forma, o segmento da população que parece ser mais afetado inclui pessoas com mais de 50 anos e pessoas com doenças crônicas pré-existentes [16]. Aproximadamente $20 \%$ dos pacientes com COVID-19 desenvolvem doença respiratória grave, com uma taxa geral de letalidade de cerca de $2,3 \%$. Pacientes com doença grave geralmente apresentam febre, tosse seca, dispneia e infiltrados pulmonares bilaterais na imagem do tórax [17].

Com isso, avaliar os efeitos de um programa de reabilitação respiratória em pacientes com COVID-19 com alta hospitalar se faz pertinente ao momento atual.

\section{Métodos}

Trata-se de uma revisão sistemática da literatura realizada através de busca digital em artigos publicados em revistas impressas e eletrônicas, ensaios clínicos, estudos randomizados e revisões sistemáticas, no período compreendido entre os anos de 2003 e 2020, nas bases de dados eletrônicas Pubmed, e Science Direct, e que apresentassem dados atuais sobre a COVID-19. Foram selecionados estudos com idioma de publicação em inglês e chinês em diferentes estratégias para assegurar uma busca abrangente. A questão norteadora deste estudo abordou como um programa de reabilitação pulmonar pode ajudar os pacientes com COVID-19 após a alta hospitalar. 
Quadro I - Descritores e operadores booleanos utilizados na busca em bases de dados

\begin{tabular}{|l|l|}
\hline Base de dados & Equaçoes de busca \\
\hline PubMed & (Rehabilitation) AND (COVID-19) AND (pulmonary) \\
\hline Science Direct & (Rehabilitation) AND (COVID-19) AND phatogenesis \\
\hline
\end{tabular}

As buscas foram realizadas por dois avaliadores independentes que selecionaram os estudos potencialmente relevantes a partir dos títulos e resumos dos resultados obtidos nas bases de dados. Quando essas seções não forneceram informações suficientes para serem incluídas, o texto completo foi verificado. Posteriormente, os mesmos revisores avaliaram independentemente os estudos completos e realizaram a seleção de acordo com os critérios de elegibilidade, ou seja, o uso de uma metodologia que tenha envolvido uma intervenção através da reabilitação pulmonar em pelo menos um grupo pesquisado. Os casos discordantes foram resolvidos por consenso.

\section{Resultados}

Na busca realizada, 101 referências foram localizadas. Na base de dados Science Direct, foram encontrados 56 artigos, 1 artigo foi selecionado para uma leitura analítica e os outros artigos não se aplicavam ao objetivo de análise ou encontravamse em duplicidade com outras bases de dados. Na base de dados PubMed foram encontrados um total de 55 artigos, sendo 15 duplicados e 31 abordavam outras técnicas ou não reportavam reabilitação pulmonar ou COVID-19. Finalmente, após a leitura analítica, 6 estudos foram selecionados como objeto de análise, por apresentarem aspectos que respondiam à questão norteadora.

\section{Discussão}

Está claro que a COVID-19 ataca principalmente o trato respiratório inferior. A pneumonia ocorre principalmente na segunda ou terceira semana de uma infecção sintomática. Sinais proeminentes de pneumonia viral incluem diminuição da saturação de oxigênio, alteração na gasometria arterial, alterações visíveis através de raios $X$ do tórax e/ou outras técnicas de imagem, com exsudatos alveolares e envolvimento interlobular, indicando eventualmente deterioração. A linfopenia parece ser comum e há aumento nos níveis de marcadores inflamatórios como proteína $C$ reativa e citocinas pró-inflamatórias [4]. 
Semelhante ao observado em resposta ao SARS-CoV, a lesão imunomediada pode desempenhar um papel crítico na patogênese da COVID-19, principalmente entre aqueles que estão gravemente doentes devido a doença grave. A infecção viral de pneumócitos induz respostas inflamatórias locais e promove a liberação de citocinas, incluindo fator de crescimento transformador-b1 (TGF-b1), fator de necrose tumoral- $\alpha$ (TNF- $\alpha$ ), interleucina-1 $\beta$ (IL-1 $\beta$ ), IL-6, bem como numerosas quimiocinas que servem para recrutar leucócitos circulantes [18]. Nas formas graves de COVID-19, a cascata inflamatória resultante pode levar a um aumento de citocinas, como observado em um estudo recente que documentou níveis séricos elevados de citocinas, incluindo IL-2, IL7, IL-10, fator estimulador de colônias de granulócitos (G-CSF), proteína quimiotática de monócitos (MCP) e TNF- $\alpha$. [19].

As consequências físicas da COVID-19 ainda não foram totalmente esclarecidas a longo prazo, mas há um consenso que os pacientes que, na fase aguda da doença, que necessitaram de ventilação mecânica podem desenvolver diversos efeitos colaterais, principalmente, a síndrome pós-cuidados intensivos [17]. Essa síndrome é caracterizada primariamente por alterações físicas, cognitivas e psiquiátricas, que têm o potencial de levar à redução da qualidade de vida dos pacientes e, muitas vezes, também de seus familiares. Neste sentido, as complexas interações entre comorbidades, complicações da doença aguda (por exemplo: hipotensão, hipóxia, hipo ou hiperglicemia e polineuromiopatia), tratamentos de suporte de vida (por exemplo: sedação, ventilação mecânica e diálise), aspectos organizacionais dos cuidados intensivos (por exemplo: restrição do contato do paciente com seus familiares) e adaptação ao período pós-UTI (por exemplo: alteração da imagem corporal, incapacidades, dificuldades de retorno ao trabalho e pobre rede de suporte social) podem contribuir para ocorrência, em longo prazo, de redução do status físico funcional, disfunção cognitiva, ansiedade, depressão e transtorno de estresse pós-traumático $[20,21]$.

Embora a COVID-19 seja nova, houve surtos anteriores de CoVSARS [17]. Em surtos anteriores, o teste da função pulmonar de 6 a 8 semanas após a alta hospitalar após a SARS mostrou padrão restritivo leve ou moderado, consistente com fraqueza muscular em $6 \%$ a $20 \%$ dos indivíduos [22]. Um estudo de coorte prospectivo de 94 sobreviventes da SARS relatou comprometimento persistente da função pulmonar em cerca de um terço dos pacientes durante 1 ano de acompanhamento. O estado de saúde desses sobreviventes da SARS também foi significativamente pior em comparação com a população saudável [23]. Um outro estudo de coorte prospectivo de 97 pacientes que sobreviveram a SARS demonstrou que $27,8 \%$ tinham achados anormais de radiografia 
de tórax, além de persistentes reduções na capacidade de exercício (teste de caminhada de 6 minutos (TC6)) em 12 meses [23].

Apesar de o COVID-19 afetar predominantemente o sistema respiratório, as evidências indicam uma doença multissistêmica que é frequentemente grave, resultando em morte. As sequelas de longo prazo da COVID-19 são desconhecidas, mas as evidências de surtos anteriores de CoV demonstram comprometimento da função pulmonar e física, redução da qualidade de vida e sofrimento emocional. Existe uma clara necessidade de orientação sobre a reabilitação dos sobreviventes do COVID19 [20].

Além da função respiratória, um estudo de coorte prospectivo de 171 sobreviventes da SARS demonstrou déficits no desempenho cardiorrespiratório (TC6min) e músculo-esquelético (dinamometria manual dos principais grupos musculares), bem como na QV em comparação com as normas da mesma faixa etária [24]. Um quadro semelhante foi relatado após a epidemia de influenza H1N1 em 2009 [25].

A reabilitação pulmonar refere-se ao tratamento individualizado de reabilitação de pacientes com doenças pulmonares crônicas após uma avaliação detalhada. Com o treinamento físico como núcleo, a reabilitação pulmonar compreende intervenções abrangentes, incluindo, entre outras, suporte psicológico e nutricional, além de mudanças educacionais e comportamentais. O objetivo da reabilitação pulmonar é não apenas melhorar as condições físicas e mentais do paciente, mas também ajudar o paciente a retornar à família e à sociedade mais rapidamente [26].

Como os pacientes com COVID-19 sofrem de vários graus de disfunção respiratória, física e psicológica, a reabilitação pulmonar é igualmente importante para pacientes admitidos e com alta para o tratamento da doença [27]. Atualmente, as evidências sobre o prognóstico de pacientes com COVID-19 são insuficientes, principalmente para pacientes idosos nos quais a doença é complicada por outras doenças. Ainda não está claro se o comprometimento de múltiplas funções sistêmicas é reversível ou se a existência prolongada do vírus pode causar disfunção física nesses pacientes. Além disso, como a COVID-19 causou uma emergência pública, os pacientes com COVID-19 podem demonstrar diferentes graus de distúrbios psicológicos, como raiva, medo, ansiedade, depressão, insônia e solidão, além de falta de cooperação e abandono de tratamento devido ao medo da doença [28].

Com base no consenso e referências, especialistas em reabilitação na China desenvolveram diretrizes práticas e viáveis de reabilitação respiratória para pacientes com COVID-19. As instruções principais dessas diretrizes são as seguintes: o objetivo a curto prazo da reabilitação pulmonar é aliviar a dispneia, a ansiedade e depressão, 
enquanto o objetivo a longo prazo é preservar a função do paciente ao máximo, melhorar sua qualidade de vida e facilitar seu retorno à sociedade [29].

No estudo de Liu et al. [30] foi utilizado o treinamento muscular respiratório, com um dispositivo de resistência manual (Threshold PEP; Philips Co) por três séries com 10 respirações em cada série; os parâmetros foram fixados em $60 \%$ da pressão expiratória máxima do indivíduo, com um período de descanso de 1 min entre os dois conjuntos. Três séries de 10 tosses ativas serão adotadas para exercícios de tosse. Para o treinamento diafragmático, cada participante irá realizar 30 contrações diafragmáticas voluntárias máximas na posição supina, colocando um peso médio (1 a $3 \mathrm{~kg}$ ) na parede abdominal anterior para resistir à descida diafragmática. Nos exercícios de alongamento, os músculos respiratórios serão alongados sob a orientação de um fisioterapeuta. Com relação aos exercícios em casa, os indivíduos serão instruídos a treinar a respiração com freno labial e a tossir, 30 séries por dia. Esta reabilitação respiratória melhora a função respiratória, a qualidade de vida e a ansiedade de pacientes com COVID-19, mas apresenta pouca melhora significativa na depressão em idosos.

A incorporação efetiva da reabilitação pulmonar no manejo da doença e na vida diária do paciente, para que ele se torne um comportamento consciente, pode fornecer benefícios a longo prazo para o paciente e sua família. Com o aprofundamento da compreensão da COVID-19, um número crescente de pacientes se recuperou. A reabilitação pulmonar para esses pacientes recuperados tornou-se um grande desafio para a equipe médica, cuja resolução requer colaboração multidisciplinar e exploração conjunta, para que seja possível fornecer suporte de alta qualidade e baseado em evidências [31].

\section{Conclusão}

A reabilitação pulmonar deve ser realizada durante todo o processo de gerenciamento de doenças, independentemente de o paciente estar hospitalizado ou em casa. Além disso, as prescrições de reabilitação devem ser individualizadas com base na condição específica do paciente. A incorporação efetiva da reabilitação pulmonar no manejo da doença e na vida diária do paciente pode fornecer benefícios a longo prazo para o paciente e sua família.

\section{Referências}


1. Sonja A. Rasmussen, MD, MS JCS. Coronavirus disease 2019 (COVID-19) and pregnancy. Responding to a rapidly evolving situation. Ann Oncol 2020;19-21. doi: 10.1016/j.ajog.2020.02.017 [Crossref]

2. Singhal T. A Review of coronavirus disease-2019 (COVID-19). Indian J Pediatr 2020;87(4):281-6. doi: 10.1007/s12098-020-03263-6 [Crossref]

3. Mahmudpour M, Keshavarz M, Farrokhi S, Nabipour I. COVID-19 cytokine storm: The anger of inflammation. Cytokine 2020. doi: 10.1016/j.cyto.2020.155151 [Crossref]

4. Velavan TP, Meyer CG. The COVID-19 epidemic. Trop Med Int Health 2020;25(3):27880. doi: 10.1111/tmi.13383 [Crossref]

5. Rothan HA, Byrareddy SN. The epidemeology and pathogensis of coronavirus (Covid19) outbreak. J Autoimmun 2020;109:1-4. doi: 10.1016/j.jaut.2020.102433 [Crossref]

6. Rothe C, Schunk M, Sothmann P, Bretzel G, Froeschl G, Wallrauch C, et al. Transmission of 2019-NCOV infection from an asymptomatic contact in Germany. N Engl J Med 2020;382(10):970-1. doi: 10.1056/NEJMc2001468 [Crossref]

7. Shi Y, Wang G, Cai X, Deng J, Zheng L, Zhu H, et al. An overview of COVID-19. 2020;21(5):343-60. doi: 10.1631/jzus.B2000083 [Crossref]

8. Xu Z, Shi L, Wang Y, Zhang J, Huang L, Zhang C, et al. Pathological findings of COVID-19 associated with acute respiratory distress syndrome, The Lancet Respiratory Medicine 2020; 8(4):420-2. doi: 10.1016/S2213-2600(20)30076-X [Crossref]

9. He F, Deng Y, Li W. Coronavirus disease 2019: What we know? 2020;(3):719-25. doi: 10.1002/jmv.25766 [ [rossref]

10. Wang D, Hu Bo, Hu Chang. Clinical characteristics of 138 hospitalized patients with 2019 novel coronavirus-infected pneumonia in Wuhan, China. Jama 2020;1-9. doi: 10.1001/jama.2020.1585 [Crossref]

11. Wu Z, McGoogan J. Characteristics of and important lessons from the coronavirus disease 2019 ( COVID-19) outbreak in China summary of a report of 72314 cases from the chinese center for disease control and prevention 2020. Jama 2019;323(13):1239-42. doi: 10.1001/jama.2020.2648 [Crossref]

12. Ramanathan K, Antognini D, Combes A, Paden M, Zakhary B, Ogino M., et al. Planning and provision of ECMO services for severe ARDS during the COVID-19 pandemic and other outbreaks of emerging infectious diseases. Lancet Respir Med 2020;8(5):518-26. doi: 10.1016/S2213-2600(20)30121-1 [Crossref]

13. Minhas AS, Scheel P, Garibaldi B, Liu G, Horton M, Jennings M, et al. Takotsubo Syndrome in the Setting of COVID-19. JACC Case Rep 2020;2(9):1321-25. doi: 10.1016/j.jaccas.2020.04.023 [Crossref]

14. Bulut C, Kato Y. Epidemiology of covid-19. Turkish J Med Sci 2020;50(SI-1):563-70. doi: 10.3906/sag-2004-172 [Crossref]

15. Guan W, Ni Z, Hu Y, Liang W, Ou C, He J, et al. Clinical characteristics of coronavirus disease 2019 in China. N Engl J Med 2020;382(18):1708-20. doi: 10.1056/NEJMoa2002032 [Crossref] 
16. Hui DS, Joynt GM, Wong KT, Gomersall CD, Li TS, Antonio G, et al. Impact of severe acute respiratory syndrome (SARS) on pulmonary function, functional capacity and quality of life in a cohort of survivors. Thorax 2005;60(5):401-9. doi:

10.1136/thx.2004.030205 [Crossref]

17. Robinson CC, Rosa RG, Kochhann R, Schneider D, Sganzerla D, Dietrich C, et al. Quality of life after intensive care unit: A multicenter cohort study protocol for assessment of long-term outcomes among intensive care survivors in Brazil. Rev Bras Ter Intensiva 2018;30(4):405-13. doi: 10.5935/0103-507x.20180063 [Crossref]

18. Razzaque MS, Taguchi T. Pulmonary fibrosis: cellular and molecular events. Pathol Int 2003;53(3):133-45. doi: 10.1046/j.1440-1827.2003.01446.x [Crossref]

19. Huang KJ, Su IJ, Theron M, Wu YC, Lai SK, Liu CC, Lei HY. An interferon-gammarelated cytokine storm in SARS patients. J Med Virol 2005;75(2):185-94. doi: 10.1002/jmv.20255 [Crossref]

20. Zhu H, Rhee JW, Cheng P, Waliany S, Chang A, Witteles RM, et al. Cardiovascular complications in patients with COVID-19: consequences of viral toxicities and host immune response. Curr Cardiol Rep 2020;22(5):32. doi: 10.1007/s11886-020-01292-3 [Crossref]

21. Berger JR. COVID-19 and the nervous system. J Neurovirol 2020. https://doi.org10.1007/s13365-020-00840-5 [Crossref]

22. Barker-Davies RM, O'Sullivan O, Senaratne KPP, Baker P, Cranley M, Dharm-Datta S, et al. The Stanford Hall consensus statement for post-COVID-19 rehabilitation. $\mathrm{Br} \mathrm{J}$ Sports Med 2020;bjsports-2020-102596. doi: 10.1136/bjsports-2020-102596 [Crossref]

23. Chan KS, Zheng JP, Mok YW, Li YM, Liu YN, Chu CM, et al. SARS: Prognosis, outcome and sequelae. Respirology 2003;8:36-40. doi: 10.1046/j.14401843.2003.00522.x [Crossref]

24. Ong KC, Ng AWK, Lee LSU, Kaw G, Kwek SK, Leow MKS, et al. 1-Year pulmonary function and health status in survivors of severe acute respiratory syndrome. Chest 2005;128(3):1393-400. doi: 10.1378/chest.128.3.1393 [Crossref]

25. Hui DS, Wong KT, Ko FW, Tam LS, Chan DP, Woo J, et al. The 1-year impact of severe acute respiratory syndrome on pulmonary function, exercise capacity, and quality of life in a cohort of survivors. Chest 2005;128(4):2247-61. doi: 10.1378/chest.128.4.2247 [Crossref]

26. Lau HMC, Lee EWC, Wong CNC, Ng GYF, Jones AYM, Hui DSC. The impact of severe acute respiratory syndrome on the physical profile and quality of life. Arch Phys Med Rehabil 2005;86(6):1134-40. doi: 10.1016/j.apmr.2004.09.025 [Crossref]

27. Vos T, Abajobir AA, Abbafati C, Abbas KM, Abate KH, Abd-Allah F, et al. Global, regional, and national incidence, prevalence, and years lived with disability for 328 diseases and injuries for 195 countries, 1990-2016: A systematic analysis for the Global Burden of Disease Study 2016. Lancet 2017;390(10100):1211-59. doi: 10.1016/S01406736(17)32154-2 [Crossref] 
28. Spruit MA, Singh SJ, Garvey C, Zu Wallack R, Nici L, Rochester C, et al. An official American thoracic society/European respiratory society statement: Key concepts and advances in pulmonary rehabilitation. Am J Respir Crit Care Med 2013;188(8). doi: 10.1164/rccm.201309-1634ST [Crossref]

29. Yang L-L, Yang T. Pulmonary rehabilitation for patients with coronavirus disease 2019 (COVID-19). Chronic Dis Transl Med 2020;6(2):79-86. doi: 10.1016/j.cdtm.2020.05.002 [Crossref]

30. Liu K, Zhang W, Yang Y, Zhang J, Li Y, Chen Y. Respiratory rehabilitation in elderly patients with COVID-19: A randomized controlled study. Complement Ther Clin Pract 2020;39:101166. doi: 10.1016/j.ctcp.2020.101166 [Crossref]

31. Heneka MT, Golenbock D, Latz E, Morgan D, Brown R. Immediate and long-term consequences of COVID-19 infections for the development of neurological disease. Alzheimers Res Ther 2020;12(1):69. doi: 10.1186/s13195-020-00640-3 [Crossref] 